Research Paper

\title{
Andrographolide Suppress Tumor Growth by Inhibiting TLR4/NF-KB Signaling Activation in Insulinoma
}

\author{
Qian-Qian Zhang ${ }^{*}$, Yi Ding ${ }^{*}$, Yan Lei ${ }^{1}$, Cui-Ling Qi1 ${ }^{1}$, Xiao-Dong He ${ }^{1}$, Tian Lan ${ }^{1}$, Jiang-Chao Li1 ${ }^{1}$ Ping \\ Gong ${ }^{1}$, Xuesong Yang ${ }^{3}$, Jian-Guo Geng ${ }^{2}$, Li-Jing Wang ${ }^{1 凶}$
}

1. Vascular Biology Research Institute, Guangdong Key Laboratory of Pharmaceutical Bioactive Substances, Guangdong Pharmaceutical University, Guangzhou 510006, China.

2. Department of Biologic and Materials Sciences, University of Michigan School of Dentistry, Ann Arbor, MI 48109, USA.

3. Department of Histology and Embryology, Key Laboratory for Regenerative Medicine of the Ministry of Education, Medical College of Jinan University, Guangzhou 510632, China.

* These authors contributed equally to this work.

$\triangle$ Corresponding author: Li-Jing Wang, phone: 86-20-39352231, fax: 86-20-39352397, wanglijing@gdpu.edu.cn; and Jian-Guo Geng, phone: 86-20-39352231, fax: 86-20-39352397, genglab@gmail.com.

(c) Ivyspring International Publisher. This is an open-access article distributed under the terms of the Creative Commons License (http://creativecommons.org/ licenses/by-nc-nd/3.0/). Reproduction is permitted for personal, noncommercial use, provided that the article is in whole, unmodified, and properly cited.

Received: 2013.09.22; Accepted: 2014.01.26; Published: 2014.03.21

\begin{abstract}
Insulinomas are rare tumors, and approximately 10\% of insulinomas are malignant. Accumulating evidence has implicated that we still lack effective therapy to treat the patients who are diagnosed with rare malignant insulinoma. Previous studies have reported that Andrographolide (Andro) could inhibit cell cycle progression, reduce cell invasion and induce cell apoptosis in many common cancer cells. However, the effects of andro are cell type-dependent. So we emplored the $\beta-T C-6$ cells and the RIPI-Tag2 transgenic mouse model of endogenously growing insulinoma model to elucidate the possible anti-cancer effect of Andro on insulinoma, an uncommon type of malignant cancers in this study. Our experiments revealed that Andro significantly inhibited tumor growth at both the early-stage and the advanced-stage of insulinoma through targeting the TLR4/NF-KB signaling pathway. This work initially provides the evidence that the TLR4/NF-KB signaling pathway might be vital as a potential therapeutic target, and also indispensable in Andro-mediated anti-cancer effect in insulinoma.
\end{abstract}

Key words: Andrographolide; insulinoma; growth.

\section{Introduction}

Insulinoma is the most common pancreatic islet cell tumor, a rare malignant tumor of the pancreas, and accounts for approximately $1.3 \%$ of pancreatic cancer [1]. Due to the late or incorrect diagnosis, the deficiency of clinical expertise \& studies and lack of access to appropriate treatments, the therapeutic strategies and anti-cancer drugs against malignant insulinoma are still limited [2]. Therefore, it is easy to understand that malignant insulinoma is becoming a challenge to clinical diagnosis and treatment. Thus, studying the molecular implications of genetic alterations underlying malignant insulinoma and exploring the effective therapeutic drugs should aid in the development of new prognostic, diagnostic and therapeutic approaches.

The RIP1-Tag2 transgenic mouse model is a well-established and well-characterized endogenously growing insulinoma model. Briefly, in the RIP1-Tag2 mice, normal islets are morphologically asymptomatic until 3 to 4 weeks of age; hyperplastic and dysplastic islets then begin to appear at 4-5 weeks of age; angiogenic islets (early-stage of tumor growth) arise from hyperplastic/dysplastic islets, and appear beginning around 6 weeks of age; tumors emerge at 
9-10 weeks as small encapsulated tumors that progress by 12 weeks into invasive carcinomas $[3,4]$. This transgenic mouse model is facilitating for study the molecular mechanism of multistep tumorigenesis and angiogenesis, and it is widely utilized in various areas of cancer research [3,4]. So we employed the mouse model to explore an efficiency drug to treat insulinoma in this study.

Andrographolide (Andro) is a diterpenoid lactone which the major bioactive component is isolated from Andrographis paniculata, a traditional herbal medicinal plant. Andro possesses multiple pharmacological activities, and it is widely employed for the treatment of inflammation and viral infections in clinic. Reports indicate that Andro could affect the NF-KB signaling, reactive oxygen species and nitric oxide synthase so that it implements a marked influence on the anti-inflammatory activity [5-7]. However, there have recently been reports that the anti-cancer effect of Andro was responding on the cell context in different cancer types. Andro can inhibiting cell cycle progression, reducing cell invasion or inducing cell apoptosis by targeting different target genes in different cancer cells $[8,9]$. Nevertheless, whether Andro can possess anti-cancer activity in the insulinoma growth environment in vivo is currently undetermined.

The TLR signaling pathway is responsible for the initiate of a cascade of events, and leads to the activation of NF-KB. Ligand binding to TLR cause the conformational change to recruit the adaptor protein MyD88 or other signaling molecules, phosphorylate and depredate I $\mathrm{K}$ to increase the phosphorylate and translocation of NF-kB to the cell nucleus, eventually bind to its target promoter region for activating transcription that play an important role in the innate immune system [10]. However, many literatures reported that either deficiency TLR4, MyD88 or NF-kB inactivation could protect tumorigenesis [11-13]. Moreover, the recent reports demonstrated that TLR4/MyD88/NF-kB signaling pathway is of vital importance in maintaining the invasive ability of pancreatic cancer cells [14], and NF-KB signaling played an important role in the development of insulinoma [15]. Therefore, it remains obscure whether or not TLR4/MyD88/NF- $\mathrm{KB}$ signaling could act as a therapeutic target in insulinoma.

In this study, $\beta$-TC- 6 cells and transgenic mouse model of spontaneous insulinoma were explored and attempted to define the effect of Andro on the treatment of insulinoma. And we treated RIP1-Tag2 mice at early-stage (angiogenic islet, 8 weeks of age) for prevention trial to detect the inhibition ability of Andro in blocking tumor development, and advanced-stage (tumor, 10 weeks of age) RIP1-Tag2 mice were treated for intervention studies to address whether Andro can slow or stop tumor growth. After being used to expose the $\beta$-TC- 6 cells and transgenic mouse model of insulinoma for prevention trial and intervention trial, Andro was found to strongly inhibit cell proliferation and tumor growth at both the earlyand advanced- stage. Furthermore, we demonstrated that Andro significantly suppressed the expression of TLR4/MyD88/NF-kB signaling pathway at protein level in RIP1-Tag2 mice. Taken together, our data revealed that Andro might possess anti-cancer activity and its anti-cancer effect was achieved by modulating the TLR4/NF-kB signaling pathway in insulinoma.

\section{Materials and methods}

\section{Reagents and Antibodies}

Andrographolide (Sigma-Aldrich) was dissolved in dimethyl sulfoxide (DMSO), and the final concentrations of DMSO were kept below $0.1 \%$ in all cell cultures and $1 \%$ in all mice. All siRNAs were obtained from RiboBio Co., Ltd. BrdU and Anti-BrdU antibody was obtained from Sigma-Aldrich. The following primary antibodies were used for western blotting: TLR4 (sc-30002), pp65 (276, sc-101749), pp65 (536, sc-101752), and pp50 (sc-101744) were obtained from Santa Cruz Biotechnology Inc.; pIkBa (\#9246) and $\beta$-actin (\# 4970) were obtained from Cell Signaling Technology; MyD88 (ab2064) was obtained from Abcam; p50 (06-886) was obtained from upstate; p65 (BA0610) and CD34 (BA0532) were obtained from BOSTER; Tunel (11684809910) was obtained from Roche.

\section{Cell Lines and Transfection}

$\beta$-TC-6 insulinoma cell line was obtained from the cell bank of Chinese Academy of Sciences (Shanghai, China). These cells were maintained as adherent cultures in Dulbecco's Modified Eagle's Medium (DMEM, GIBCO), supplemented with 20\% fetal bovine serum (FBS), $100 \mathrm{U} / \mathrm{mL}$ penicillin, and $100 \mu \mathrm{g} / \mathrm{mL}$ streptomycin, and was incubated at $37^{\circ} \mathrm{C}$ in a humidified chamber supplemented with $5 \% \mathrm{CO}_{2}$. Cells transfection with siRNAs was performed using Lipofectamine 2000 (Invitrogen). All RNA transfections were performed at a final concentration of 50 $\mathrm{nM}$.

\section{Animal manipulations}

RIP1-Tag2 mice were obtained from the Jackson Laboratory (Bar. Harbor, Maine); C57BL/6J mice were obtained from Guangdong Medical Laboratory Animal Center. All animals involved in experiments were treated in accordance with the "Guide for the Care and Use of Laboratory Animals" by the National Academy of Sciences (NIH publications No. 80-23, 
revised 1996). According to the the $\mathrm{LD}_{50}$ for intraperitoneally administered andrographolide (11.6 g/ kg) [16], RIP1-Tag2 mice were randomized into 3 groups (6 mice per group): untreated group (Mock) or intraperitoneal injection with $5 \mu \mathrm{g} / \mathrm{g}$ DMSO or Andro twice a week for 4 weeks at 8 weeks (early-stage of cancer, prevention trial) or 10 weeks (advanced-stage of cancer, intervention trial) after birth, and BrdU was intraperitoneal injected into the mice 1.5 hours before sacrificed. Then the tumor volume and number were detected. Tumor volume (V) was monitored by measuring the length $(\mathrm{L})$ and width $(\mathrm{W})$ with calipers, and eventually calculated with the formula $\left(L \times W^{2}\right)$ $\times 0.5236$. Tumor burden of per mouse was calculated as the total volume of tumors of each mouse.

\section{Cell growth and proliferation assay}

Cells were plated in each well of a 96-well plate. The indicated concentration of Andro was added 24 hours later, and the measurement was performed at the indicated times. Cell viability was evaluated with MTT assay.

Cell proliferation was assessed with colony formation assay. The 4,000 cells were seeded in 6-well plates and $10 \mu \mathrm{M}$ Andro was added 24 hours later for 7 days to form colonies. The colonies were fixed in $4 \%$ paraformaldehyde and stained in a $0.1 \%$ crystal violet solution.

\section{Hoechst 33342 Staining and Evaluation}

Cells $\left(5 \times 10^{5}\right.$ cells/well) were plated in 6-well plates and treated with $\mathrm{IC}_{50}$ concentrations of Andro or DMSO for $48 \mathrm{~h}$. Then the Hoechest $33342(5 \mathrm{mg} / \mathrm{ml}$, Beyotime) staining and evaluation were carried out as indicated before [17].

\section{Histologic, Immunohistologic and Immunoblot Analysis}

Tumor tissues in mice were fixed in 10\% neutral formalin solution for 24 hours and followed by paraffin embedding. Then paraffin sections $(4 \mu \mathrm{m})$ were stained with hematoxylin \& eosin (H\&E) or antibodies. Hematoxylin \& eosin (H\&E) and immunohistochemical staining for bromodeoxyuridine (BrdU), TUNEL, CD34, TLR4, MyD88, pp65, p50 and p65 were performed as previously described [18].

Total protein were prepared using RIPA buffer indicated previously, and analyzed by western blotting as previously described [19].

\section{Microvessel Density}

Microvessel density (MVD) was recorded as the number of point counts of CD34-positive vessels per field at $\times 40$. Ten fields were randomly selected in a section from nonnecrotic areas of tumors were examined. Microvessel density counts were recorded in- dependently by two observers in sections from three mice of each group.

\section{Statistical Analysis}

Data are presented as the mean \pm standard deviation from at least 3 separate experiments. Unless otherwise noted, the differences between groups were analyzed using a Student's $t$ test when only two groups were compared. All tests were two-sided. Differences were considered statistically significant at $P<0.05$. The proteins expression level in the immunohistochemical slices was determined by measuring cumulated integrated optical density (IOD) using IPP software.

\section{Results}

\section{Andrographolide suppresses insulinoma tumor growth in vivo}

Andro (Fig. 1A) is the major bioactive component isolated from Andrographis paniculata, and it is widely used for the treatment of inflammation and viral infections in clinic. Recently reports indicated that Andro possess anti-cancer activity. However, whether Andro can inhibit tumor growth in insulinoma is currently undetermined. To evaluate the potential effect of Andro on insulinoma tumorigenesis, Andro was exposed to the genetically engineered mouse models of insulinoma (RIP1-Tag2 mice) to examine the effect on tumor growth. For prevention trial and intervention trial, the 8-week old (early-stage of cancer) and 10-week old (advanced-stage of cancer) RIP1-Tag2 mice were treated with $5 \mu \mathrm{g} / \mathrm{g}$ DMSO or Andro twice a week for 4 weeks. Then we detected the effect on tumor growth of Andro in RIP1-Tag2 mice. The tumor number and tumor burden of per mouse were significantly suppressed by Andro compared with control groups (Fig. 1B and 1C). Therefore, the in vivo studies suggest that Andro significantly inhibits tumorigenicity of insulinoma both in the early-stage and in the advanced-stage.

\section{Andrographolide inhibits cell proliferation and angiogenesis in insulinoma}

To further elucidate the potential regulatory mechanism of Andro on tumor growth, we detected the cell proliferation and angiogenesis using immunohistochemical staining. The results showed that Andro can decrease BrdU staining of DNA incorporation, which is a marker for cell proliferation, in RIP1-Tag2 mice (Fig. 2A). But whether the tumor cells were undergoing apoptosis or not was not known. TUNEL (terminal deoxynucleotidyl transferase (TdT)-mediated dUTP nick end labeling) staining was carried out to detect apoptosis in tumor tissues of RIP1-Tag2 mice (untreated group), DMSO-treated 
RIP1-Tag2 mice (control group) and Andro-treated RIP1-Tag2 mice. As shown in Fig. 2B the TUNEL-positive cells were significantly increased in

A

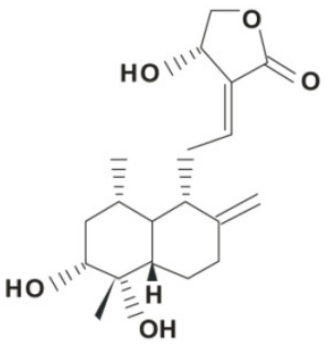

B

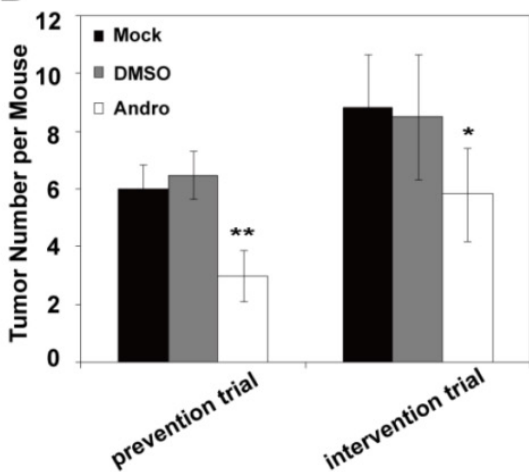

C

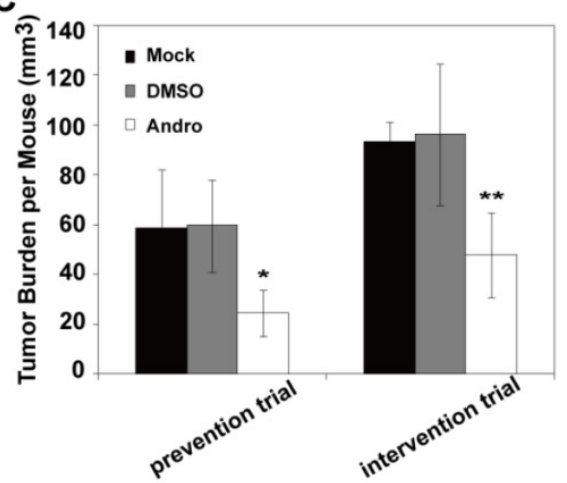

Figure I. Andro inhibits insulinoma tumor growth in vivo. (A) The structures of Andro. Andro was significantly suppresses tumor number (B) and tumor burden $(C)$ both for prevention trial (treated at 8 weeks after birth) and intervention trial (treated at 10 weeks after birth)compared with untreated group and DMSO treated group in RIPI-Tag2 mice. $\mathrm{n}=6, * P<0.05$, $* * P<0.0$ I.

A

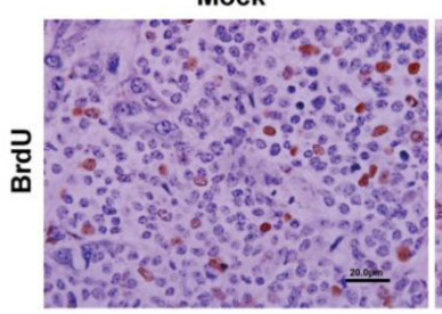

DMSO

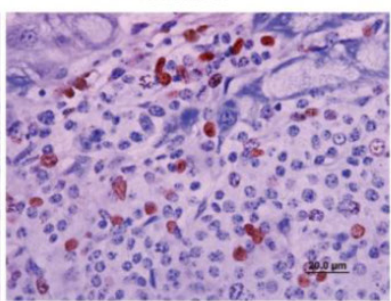

tumor tissues of Andro-treated RIP1-Tag2 mice compared with control groups.

Andro

B

Mock

DMSO
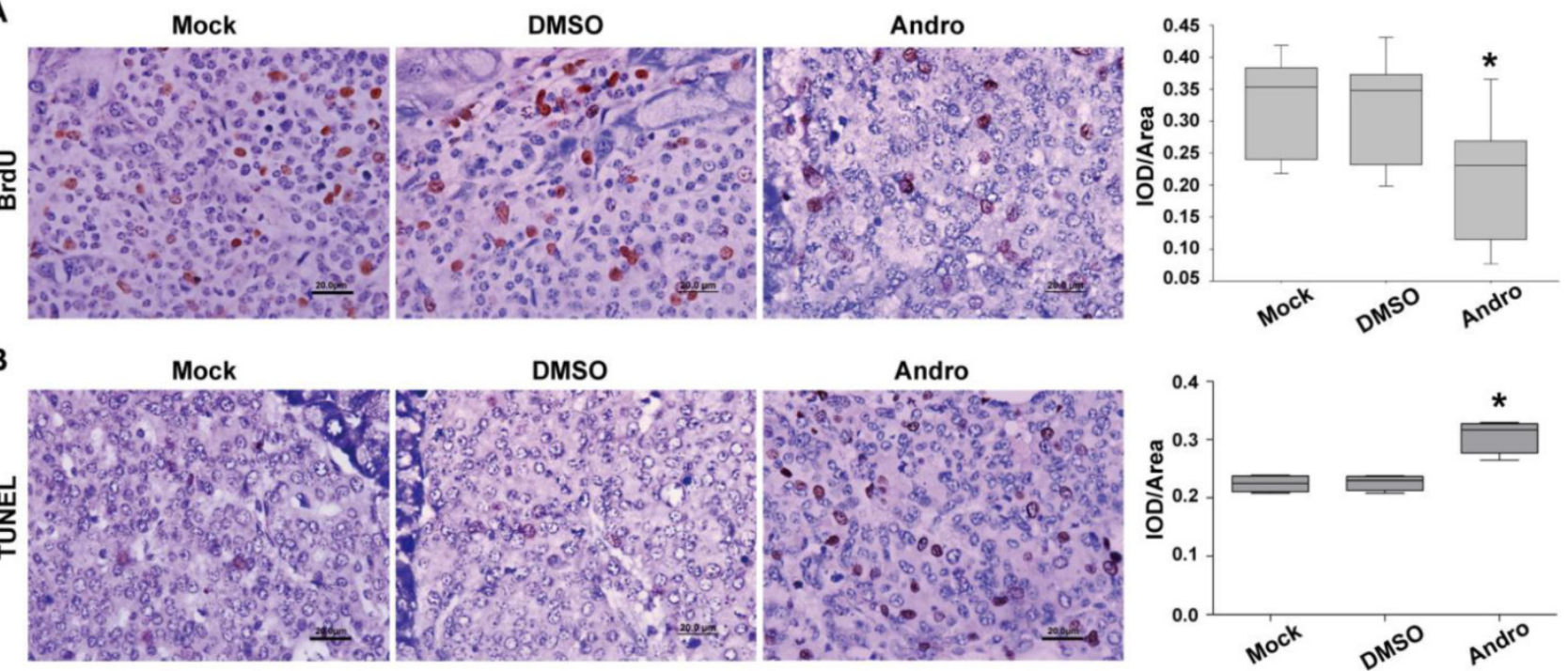

C

Mock

DMSO

Andro
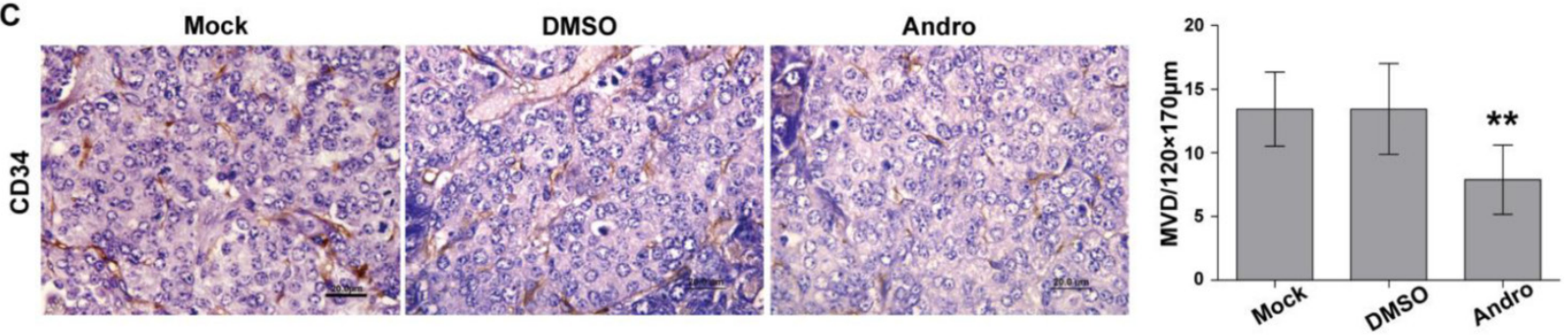

D

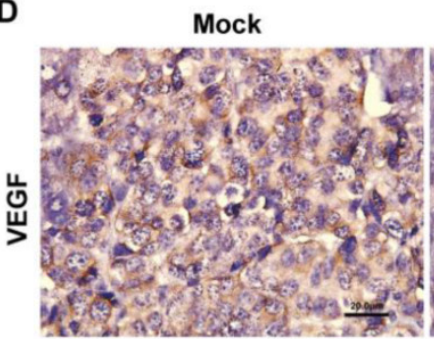

DMSO
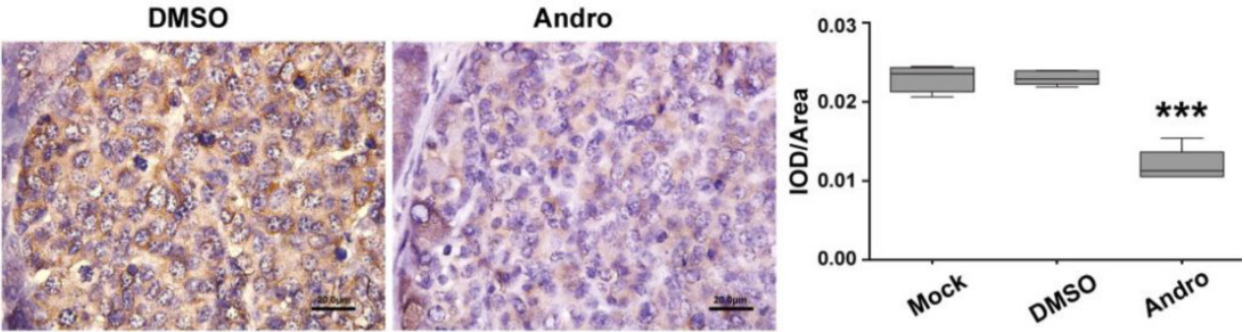

Figure 2. Andro inhibits cell proliferation and angiogenesis and induces cell apoptosis in insulinoma. (A) Andro inhibited the tumor cell proliferation, which was measured using the BrdU cell proliferation assay, in RIPI-Tag2 mice. (B) Cell apoptosis was increased, which was examined through TUNEL staining, in Andro treated tumor tissue compared with control groups. (C) and (D) Andro suppressed the tumor angiogenesis, which was measured using the immunohistochemical staining of CD34 and VEGF, in RIPI-Tag2 mice. $n=6, * P<0.05, * * P<0.01$. Bar, $20 \mu \mathrm{m}$. 
Meanwhile, CD34 staining, an endothelial marker, and VEGF staining were used to detect tumor angiogenesis, we found that Andro could strongly decreased CD34 positive cells and inhibited the expression of VEGF in RIP1-Tag2 tumor tissues compared with control groups (Fig. 2C and 2D). These results suggest that Andro can inhibit angiogenesis in insulinoma. All the data showed that Andro could significantly suppress tumor growth through inhibiting cell proliferation and angiogenesis, and promoting apoptosis of tumor cells in insulinoma.

\section{Andrographolide suppresses cell proliferation and evoke cell apoptosis in vitro}

To further evaluate the potential effect of Andro on tumorigenesis, Andro was exposed to the pre-cultured $\beta$-TC- 6 cells to examine the Andro effect on cell proliferation. Compared with untreated cells and the exposure of DMSO, which was employed as a negative control, Andro can significantly suppressed the viability of $\beta$-TC- 6 cells in a dose- and time-dependent manner with an $\mathrm{IC}_{50}$ of about $10 \mu \mathrm{M}$ for 48-hour treatment (Fig. 3A and 3B). Meanwhile, the cells were seeded to grow at very low density and then treated with $10 \mu \mathrm{M}$ Andro. As shown in Fig. 3C, the colonies treated with Andro were obviously lesser and smaller than those in the control groups. These results suggest that Andro could significantly suppress cell proliferation of $\beta$-TC- 6 cells through targeting some oncogenes. Meanwhile, we detected the apoptosis using hochest 33342 dye in Andro treated $\beta$-TC- 6 cells and found that Andro can markedly induced cell apoptosis. As depicted in Fig. 3D, cells treated with $10 \mu \mathrm{M}\left(\mathrm{IC}_{50}\right)$ Andro presented morphological characteristics of apoptosis were observed. Compared with control group, chromatin condensation and DNA fragmentation were found in Andro-treated $\beta$-TC- 6 cells. Therefore, both in vivo and in vitro studies suggest that Andro significantly inhibits tumorigenicity by inhibit cell proliferation and induce cell apoptosis.

\section{The expression and activation of TLR4/NF-KB signaling was increased during insulinoma development}

Previous studies have shown that Andro can inhibit inflammation through preventing NF-KB binding to the promoters of the target genes [5]. However, recent reports demonstrated that NF-KB activation played an important role in the development of insulinoma [15]. Moreover, it was reported that TLR4/MyD88/NF-kB signaling pathway is of vital importance in maintaining the invasive ability of pancreatic cancer cells [14] and plays a significant role in tumorigenesis and development. Nevertheless, the functional targets of Andro for suppressing tumor growth in RIP1-Tag2 mice were not yet known.

We defined the pathological process of RIP1-Tag2 with H\&E stain and classified into the stages of normal, hyperlasia, angiogeneic islet, insulinoma and invasive carcinoma as previously described (Fig. 4A) [20]. Meanwhile, we displayed here that the expression and activation of TLR/NF-KB signaling pathway, by immunohistochemical staining. p50 and p65 are two subunits of NF- $\mathrm{kB}$, the Ser276 site of p65 is phosphorylated during NF-kB activation. We examined total p50, p65 and phosphorylation p65 (Ser276) at various stages of insulinoma to detect the expression and activation of NF-KB. As shown in Fig. 4B, the p50 and p65 proteins were increased expression in the cytoplasm and the phosphorylation p65 (Ser276) protein was increased expression in the nucleus during insulinoma development. Meanwhile, the expression of TLR4 and MyD88 was also increased during insulinoma development. All the results indicated that the expression and activation of TLR/NF-kB signaling was increased during the insulinoma development in RIP1-Tag2 mice.

\section{TLR4/NF-KB signaling pathway is the target of andrographolide}

To confirm whether TLR4/NF-kB signaling pathway was the functional target of Andro, we analyzed the expression of TLR4/NF-kB signaling pathway at protein level in tumor tissues of RIP1-Tag2 mice treated with Andro. The data of western blotting indicated that Andro dramatically inhibited the expression of TLR4, MyD88, the phosphorylation of IкBa, p65, one subunit of NF-kB, and phosphorylation of p65 in tumor tissues of RIP1-Tag2 mice (Fig. 5A). As shown in Fig. 5B, Andro can significantly inhibited the expression of TLR4, MyD88 and p65 using the immunohistochemical staining in tumor tissues of RIP1-Tag2 mice. The results indicated above showed that Andro can significantly inhibited tumor growth through targeting TLR4/NF-KB signaling and suppress the phosphorylation of $\mathrm{p} 65$ subunit, rather than p50, in insulinoma.

\section{TLR4/NF-KB signaling pathway is the dominant functional target of andrographolide.}

To confirm the primary target for Andro in insulinoma, we detected the expression of TLR4, MyD88 and p65 in the cells treatment with siRNAs of these genes. As shown in Fig. 6A and 6C, TLR4 deficient could only slightly inhibited the expression of p65 and cannot suppressed the expression of MyD88, but p65 deficient could inhibited the expression of 
MyD88 but not TLR4. Moreover, the inhibition of MyD88 can also reduce the expression of p65 but not TLR4 (Fig. 6B). All the results demonstrated that TLR4 and p65 may both the primary target for Andro.

However, whether the TLR4/NF-KB signaling was the functional target of Andro was not clarified. The results indicated that, the inhibition of TLR4,
MyD88 and p65 can both suppressed cell proliferation. However, the inhibition rate of cell proliferation of MyD88 and p65 was similar and higher than that of TLR4 (Fig. 6D). The results were further demonstrated that Andro can inhibit tumor growth through targeting both TLR4 and NF-KB.

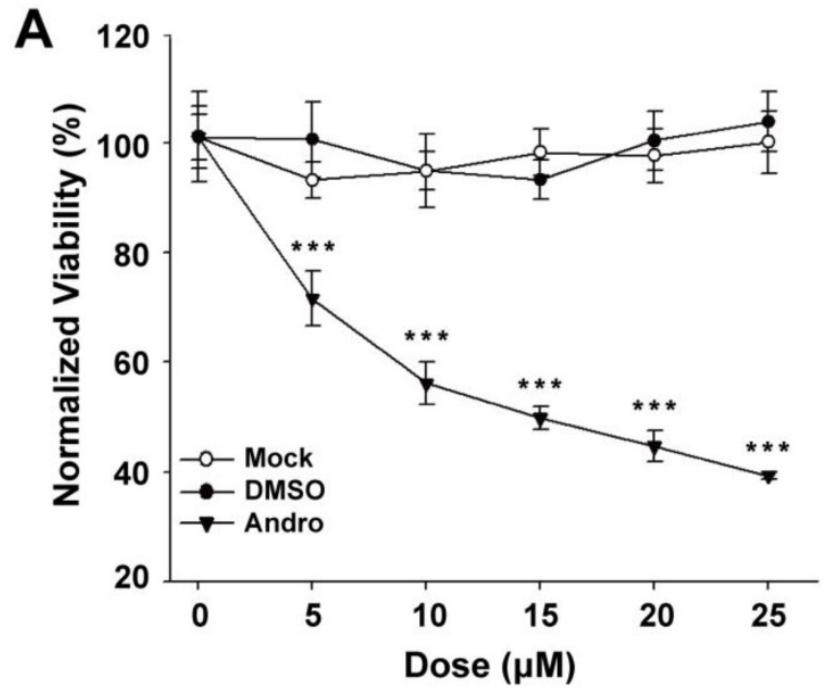

B

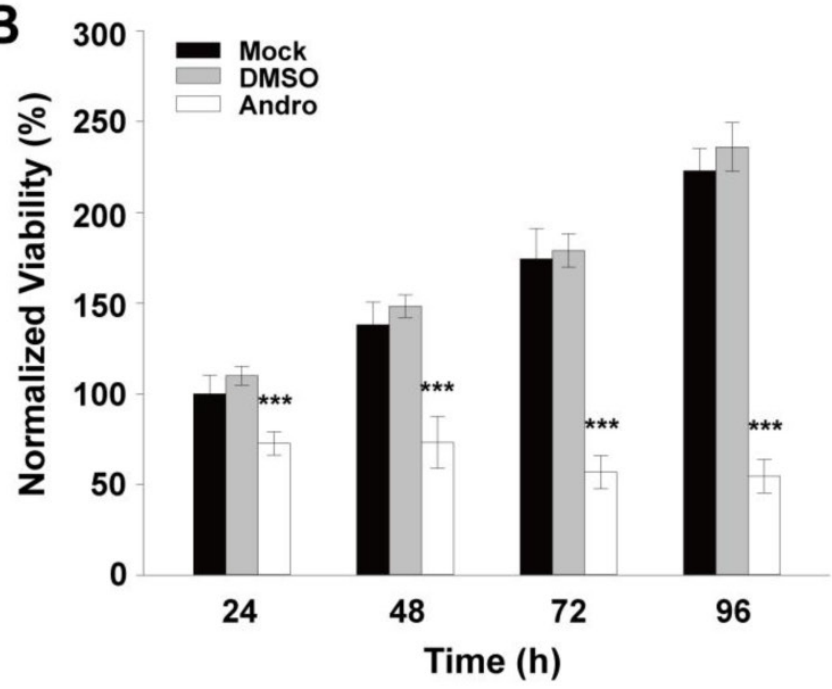

C

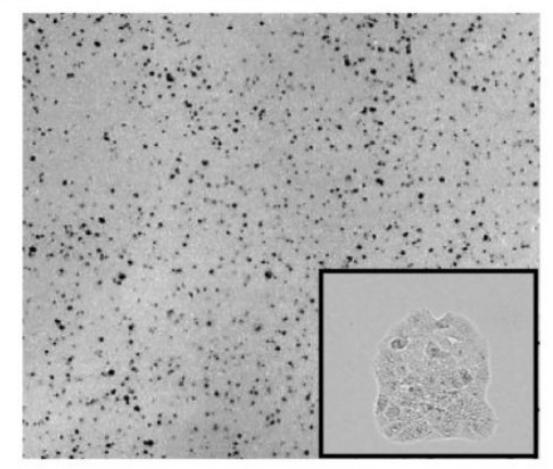

D

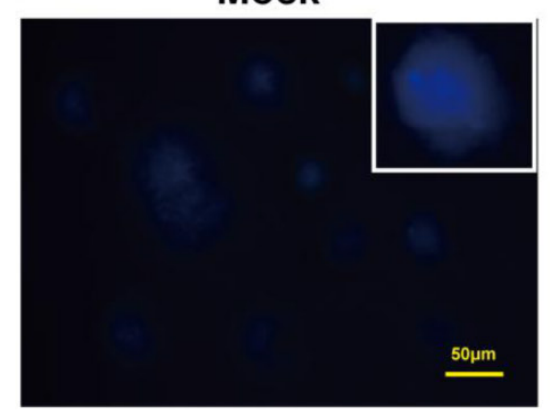

DMSO

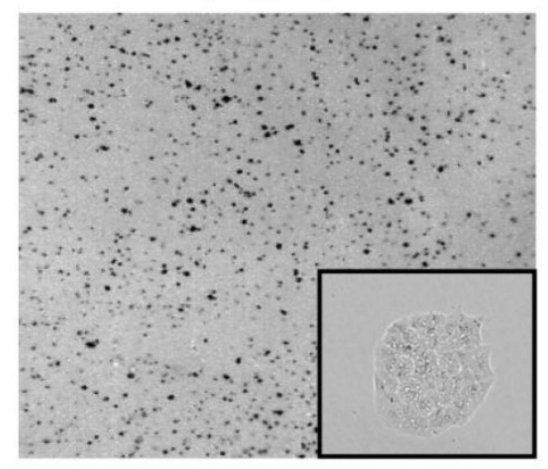

DMSO

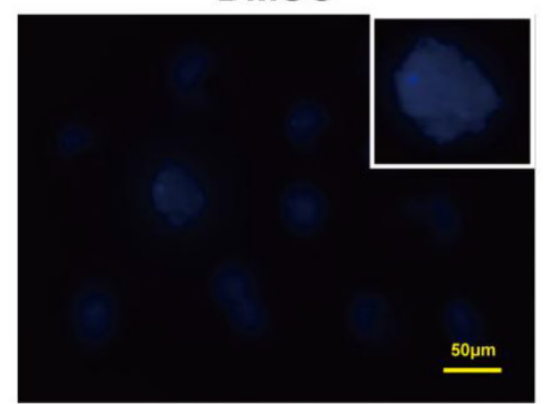

Andro

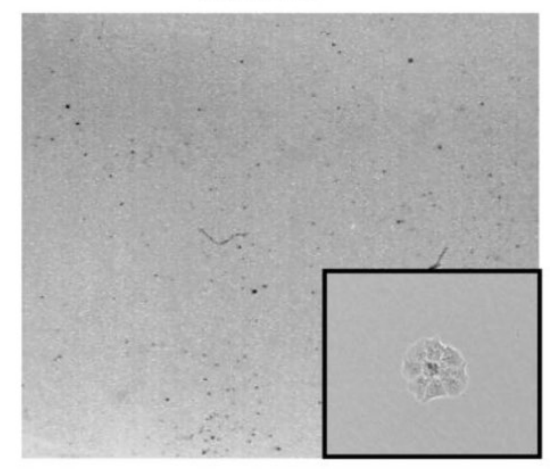

Andro

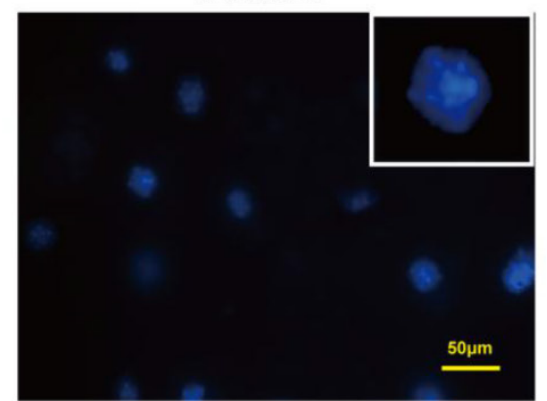

Figure 3. Andro suppresses cell proliferation and clonogenicity and induces cell apoptosis in $\boldsymbol{\beta}$-TC-6 cells. (A) The cells were treated with various doses of Andro for 48 hours, and the cell viability was quantified by MTT assay. (B) The cells were treated with the IC $C_{50}$ dose of Andro, and relative cell viability was measured using the MTT assay at the indicated times. (C) The clonogenicity ability of $\beta$-TC-6 was significantly suppressed by Andro compared with control groups. Representative photomicrographs are shown. (D) Andro induces cell apoptosis in $\beta$-TC-6 cells that treated with the IC 50 dose of Andro for $48 \mathrm{~h}$. Cells were stained with Hoechst 33342 and apoptotic cells were identified by condensation and fragmentation of nuclei using inverted light microscope. All values are presented for the mean \pm s.d., $n=I 2 ; * * * p<0.001$. 

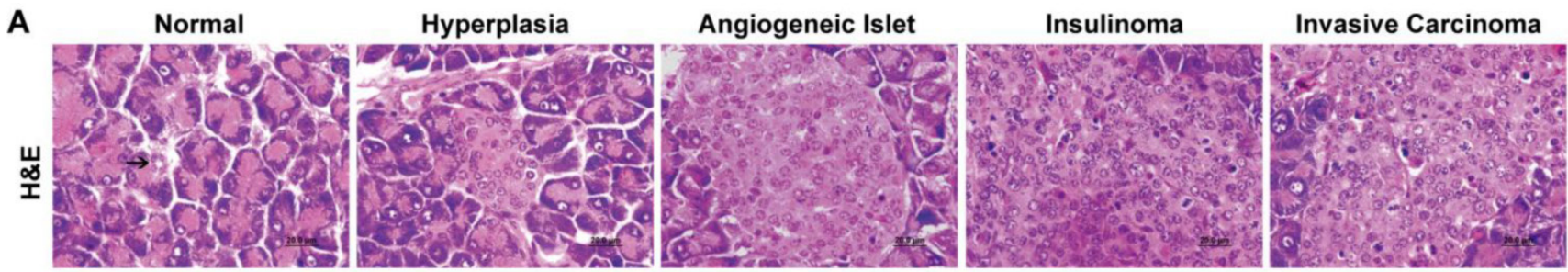

B
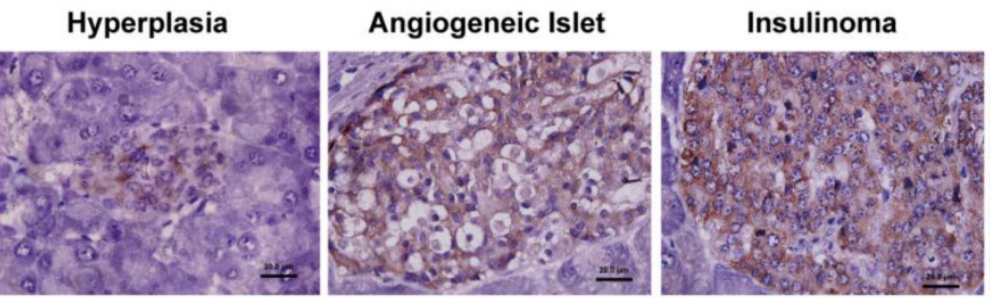

Invasive Carcinoma
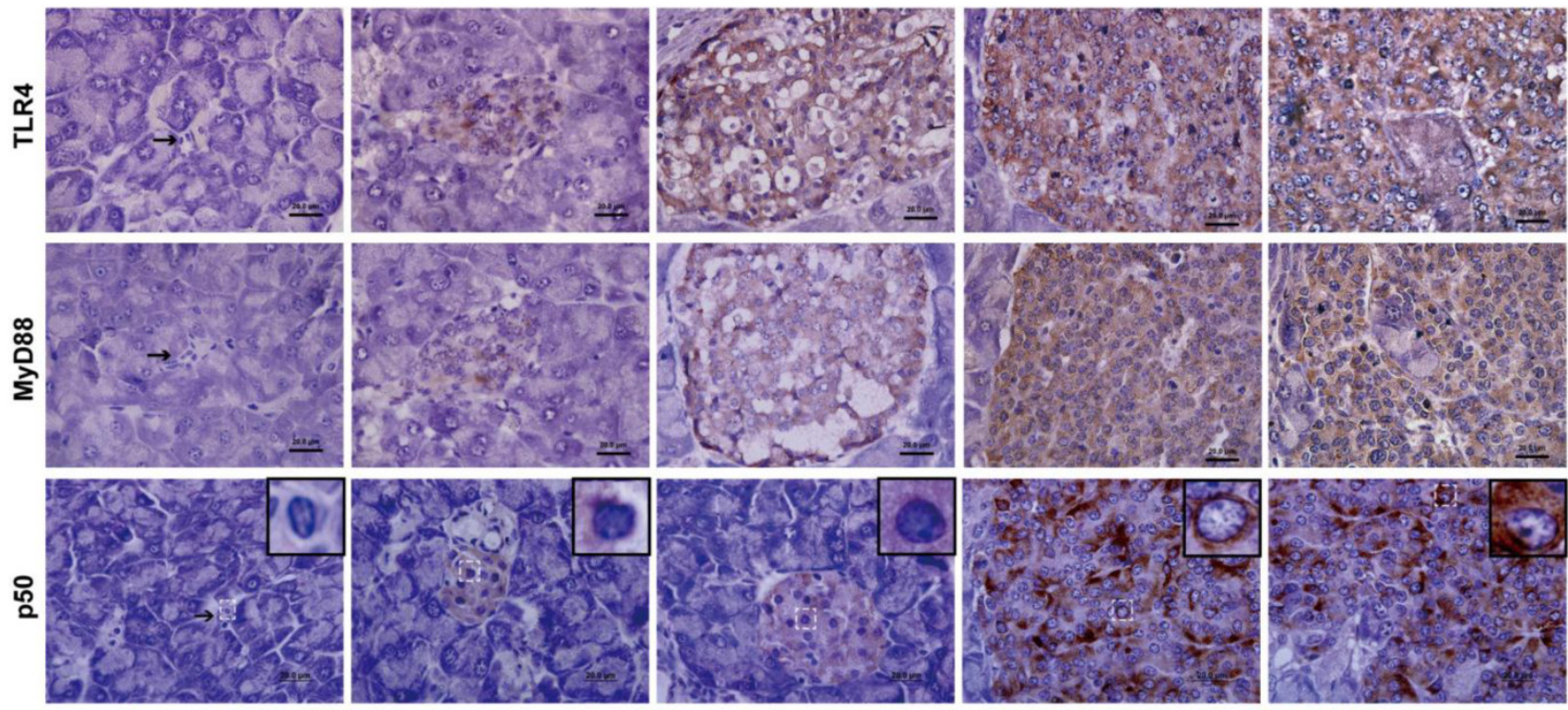

ڤ్ ڤ
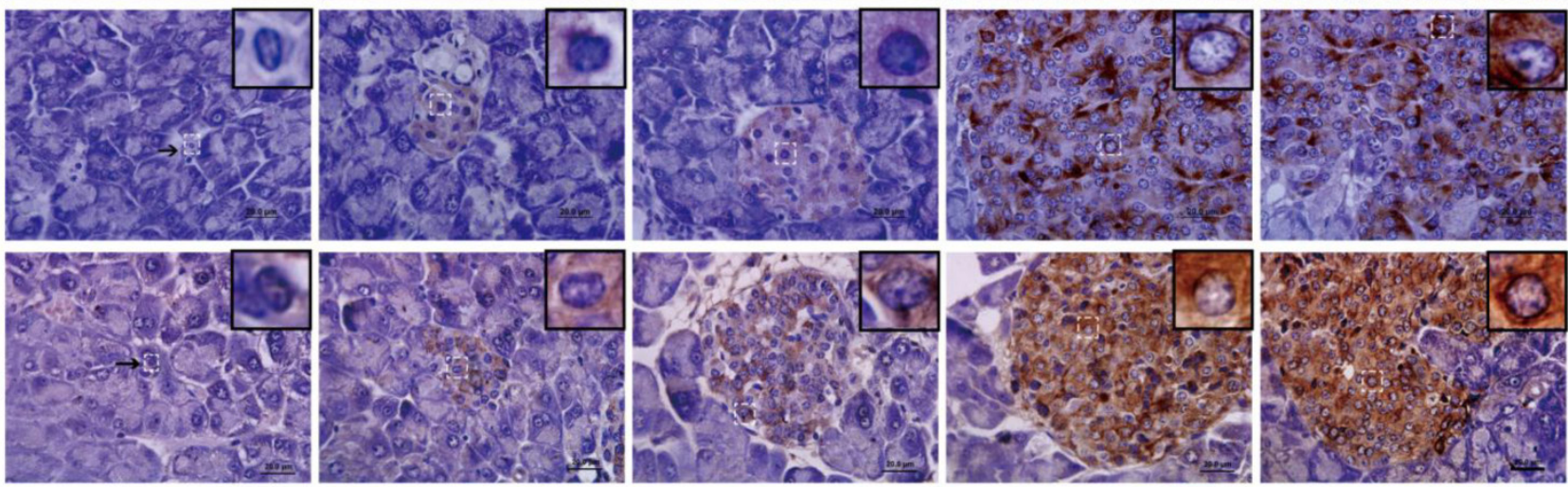

๕
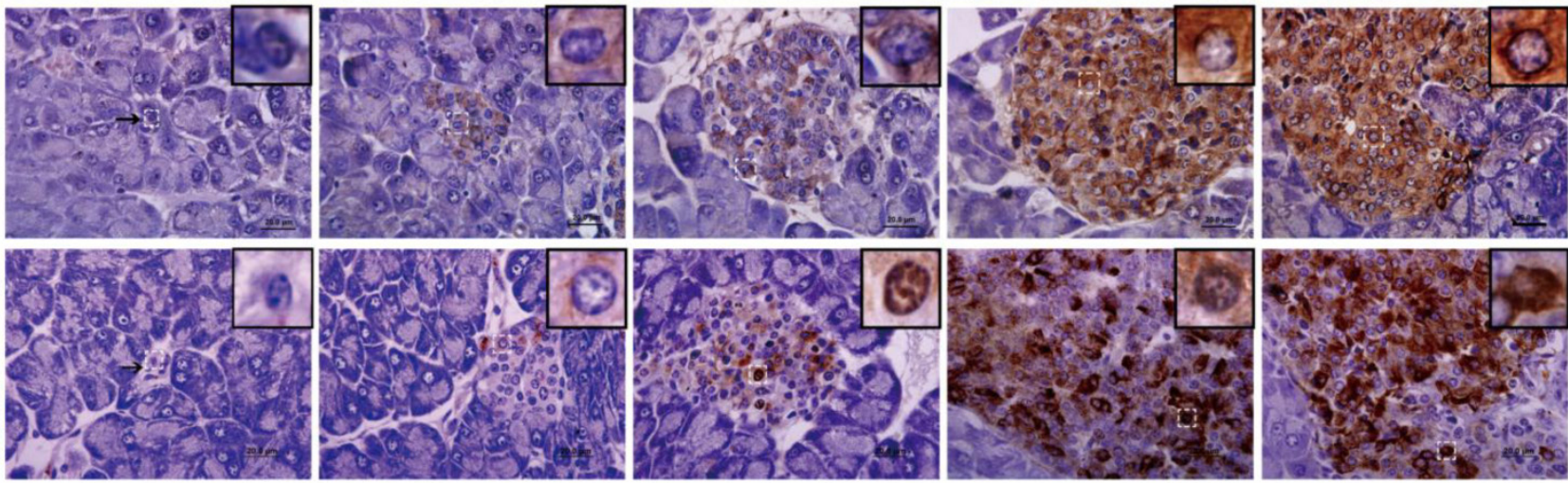

Figure 4. The expression and activation of TLR4/NF-KB signaling is increased during insulinoma development. (A) The classification of insulinoma. The H\&E staining defined the stages of insulinoma. (B) The immunohistochemical staining of TLR4, MyD88, p50, p65 and phosphorylated p65 (Ser276) at the stage of normal, hyperplasia, angiogenic islet, insulinoma, and invasive carcinoma in RIPI-Tag2 mice. Results are representative of at least 3 tissue samples in a mouse from more than 3 mice for each stage. Bar, $20 \mu \mathrm{m}$. 
A
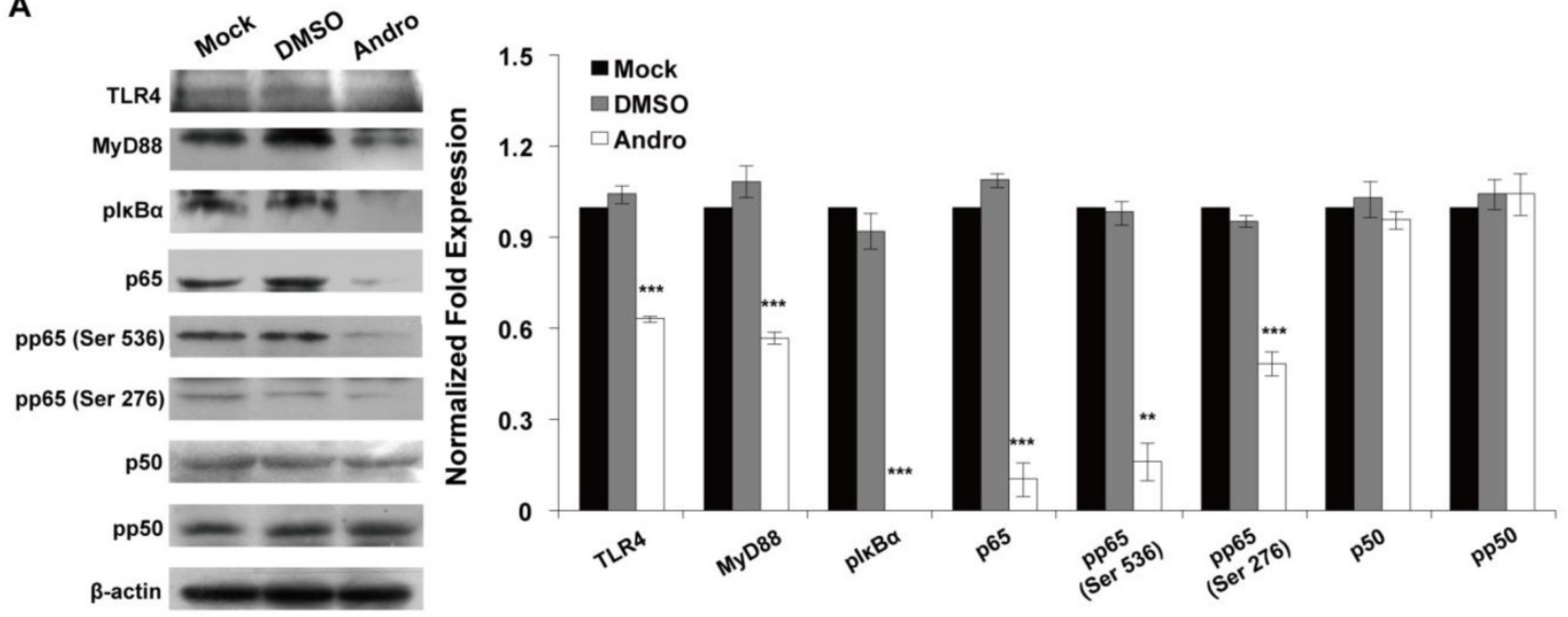

B

Mock

DMSO

Andro
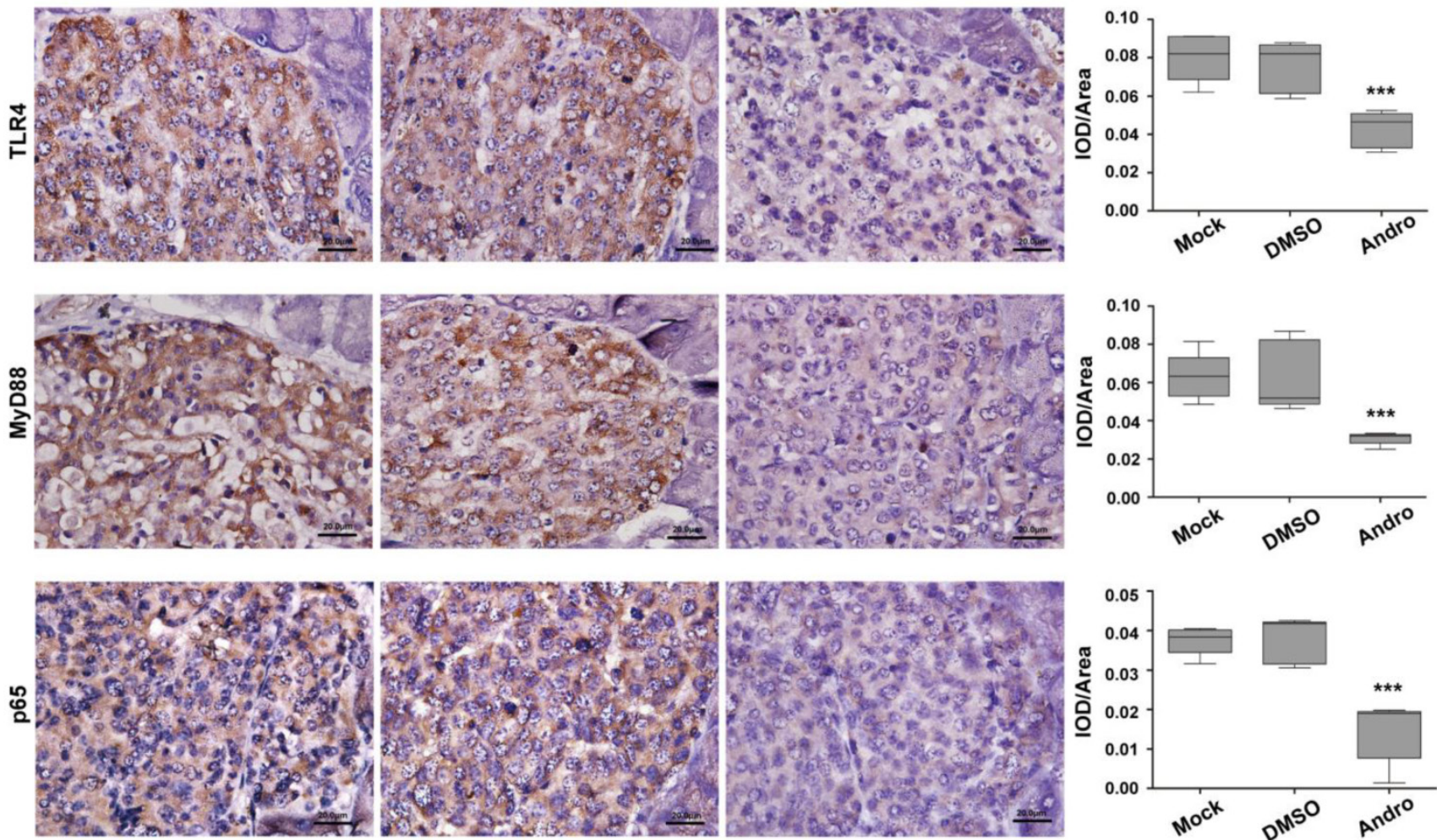

Figure 5. Andro targets TLR4/NF-KB signaling in insulinoma. Andro can significantly inhibited the protein expression of TLR4, MyD88, phosphorylated IKBa, p65, phosphorylated p65 (Ser536), and phosphorylated p65 (Ser276) of TLR4/NF-KB signaling pathway in tumor tissues of RIPI-Tag2 mice compared with control groups. Immunohistochemical staining showed that Andro also inhibited the expression of TLR4 (B), MyD88 (C) and p65 (D) in tumor tissues of RIPI-Tag2 mice compared with control groups. Bar, $20 \mu \mathrm{m}$. $* * * P<0.00 \mathrm{I}$. 

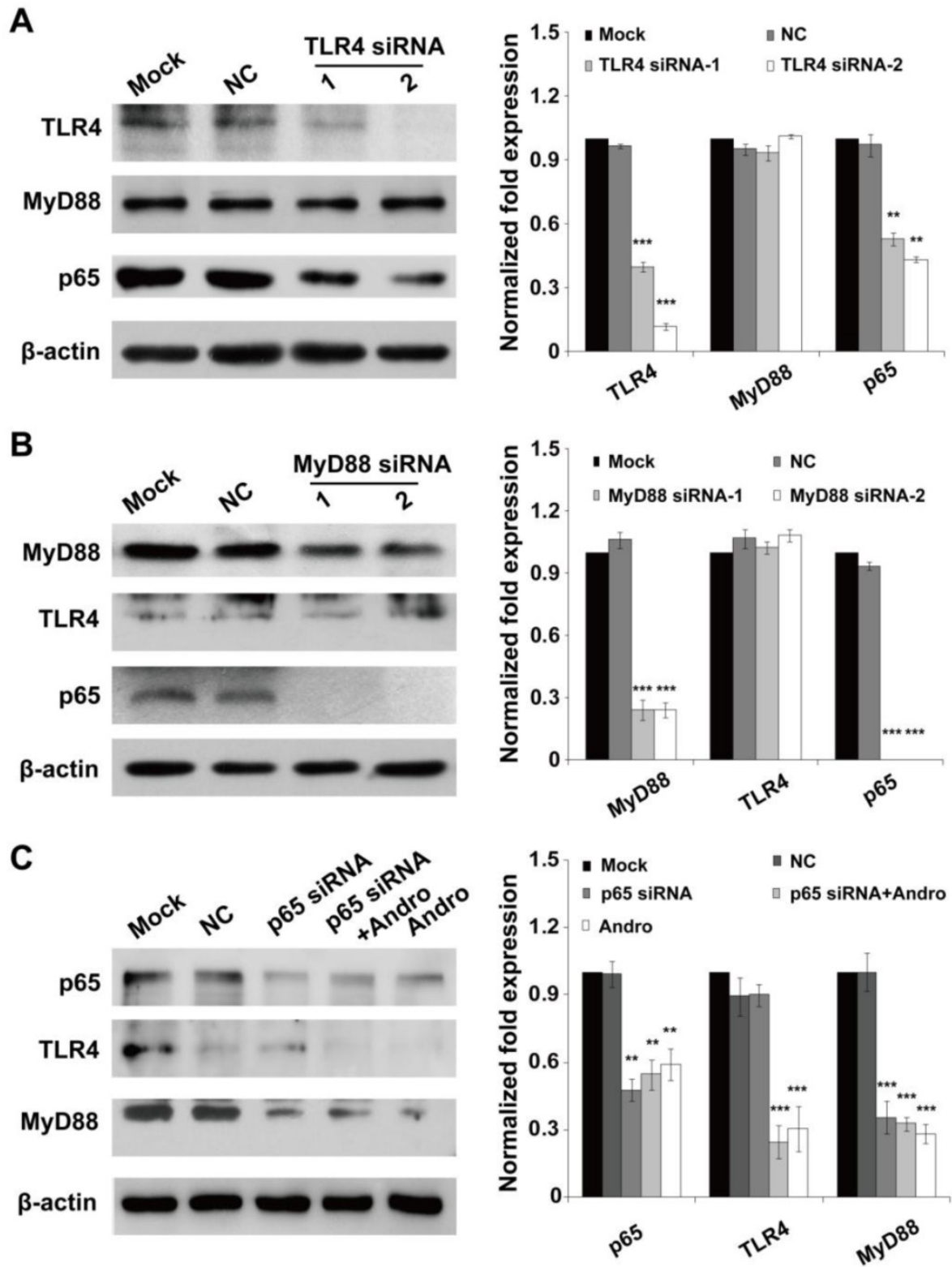

D

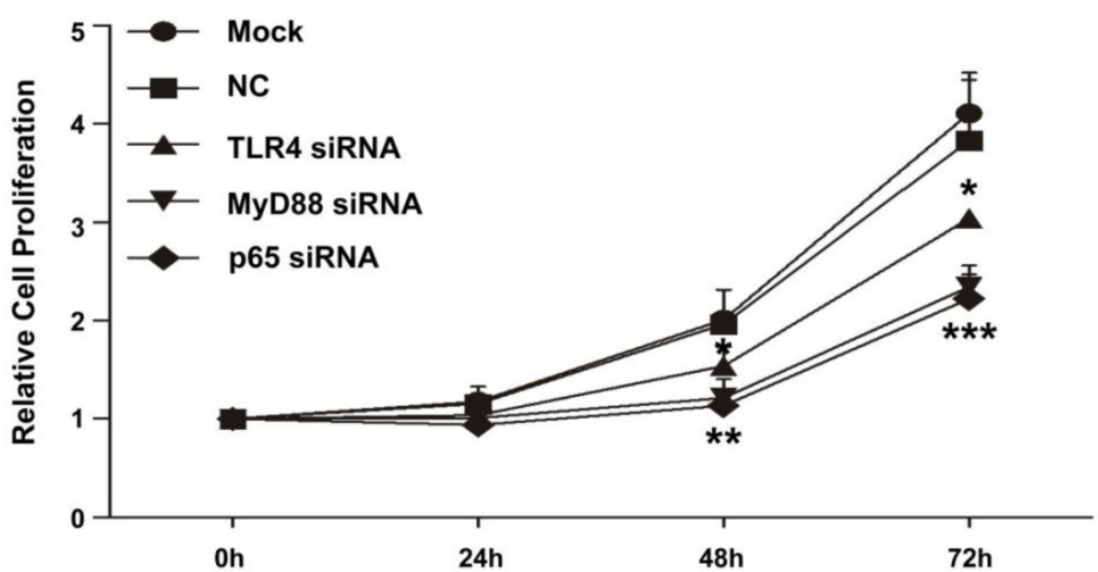

Figure 6. TLR4/NF-KB signaling pathway is the dominant functional target of andrographolide. (A) TLR4 deficient could only slightly inhibit the expression of p65 and cannot suppress the expression of MyD88. (B) The inhibition of MyD88 result the reduction of p65 but not TLR4 (C) p65 deficient could inhibit the expression of MyD88 but not TLR4. (D) The inhibition of TLR4, MyD88 and p65 can both suppressed cell proliferation. $n=12$, * $P<0.05$, ** $P<0.01$ and $* * * P<0.001$. 


\section{Discussion}

This study demonstrates that Andro acts as an anti-tumor drug against insulinoma by inhibiting tumor growth, likely through targeting TLR4/NF-kB signaling pathway. These findings support the model that TLR4 is up-regulated during insulinoma progression, directly resulting phosphorylate IKBa and increase NF-kB activity to diffuse into the cell nucleus and activate the transcription of genes to promote the cell proliferation. Meanwhile, a positive feedback loop is existent between NF- $\mathrm{kB}$ and MyD88. Interestingly, Andro can suppress the abnormal activation of TLR4/MyD88/NF-kB signaling to supply a potential new therapy to the treatment of insulinoma.

During the cancer biology studies, multiple mouse models, including xenograft, transplantable and genetically engineered mouse tumor models, have been widely used in various areas of the cancer research [21-23]. However, xenograft and transplantable mouse models are usually derived from advanced cancer cells or tissues and growth in a host with an impaired immune system that can not represent the procedure of tumorigenesis [23]. RIP1-Tag2 genetically engineered spontaneous insulinoma model is constructed using the transgenic expression of SV40 T antigens from the RIP, and lead to generate the spontaneous insulinoma that similar in morphology, histopathology and molecular characteristics to human tumor [24]. The RIP1-Tag2 mouse can mimic the process of tumorigenesis, as tumors in a host physiological condition that immune fuction, angiogenesis, and inflammatory processes can all interact normally with the developing tumor. Therefore, using the RIP1-Tag2 mouse is easy to study the regulatory mechanism of tumors early and late in the process. In this study, we chose the RIP1-Tag2 genetically engineered spontaneous insulinoma models to monitor the anticancer efficacy and mechanism of action of Andro in tumor early- and late- stage in the process.

Andrographolide (Andro) is the major active constituent of Andrographis paniculata, which has been used as an herbal medicine. It can be effectively applied to treat inflammation and infection in clinic. Recently, it has been reported that Andro has another pharmacological effect on inhibiting various common tumors cell proliferation and invasion, including hepatocellular carcinoma, cervical carcinoma, prostatic adenocarcinoma, oral squamous cell carcinoma, gastric cancer, and colon cancer [8, 9, 25-27]. But the cellular responding of Andro was dependent on the biochemical and physiological context in different cancer types. So, whether or not Andro also inhibit the tumor growth in insulinoma was still in the dimness. In this study, we demonstrated that Andro can significantly inhibit tumor growth in insulinoma. Fur- thermore, the in vivo studies also indicated that Andro can therapy of insulinoma both in the early-stage and the advanced-stage of tumors. All of our results suggesting that immunomodulatory and anti-cancer activity of Andro may be potentially used as a broad-spectrum anti-tumor drug in clinical practice.

It has been known that the effects of Andro are cell type-dependent. Andro possess anti-inflammatory activity through NF-KB inactivation [5], and Andro also inhibits tumor cell proliferation and invasion, or induces cell apoptosis by regulating the expression of p27, CDK4, and Bcl-2 family members [8, 9]. TLR4/MyD88/NF-kB signaling pathway plays a vital role in the innate immune system. TLR signaling induces immune responses through MyD88, an adaptor molecule of TLR, leading to the subsequent downstream activation of NF- $\mathrm{kB}$, and eventually inducing the expression of proinflammatory cytokines and chemokines. In addition to inflammation, TLR4/MyD88/NF-kB signaling pathway also mediates cell proliferation and invasion in ovarian cancer, pancreatic cancer, and epithelial ovarian cancer cells $[14,28,29]$. The inflammatory environment can fosters tumor growth, angiogenesis and metastasis. Researches indicated that TLR4/MyD88/NF-KB signaling pathway may plays a significant role in connecting inflammation and tumorigenesis and development. Reports indicated that insulinoma are strongly associated with the inflammatory process [20]. However, the precise regulatory mechanism of this inflammation-related pathway in insulinoma remains poorly understood. Here, we demonstrated that TLR4/MyD88/NF-kB signaling pathway may play an important role in Andro-mediated inhibiting of tumor growth in insulinoma.

\section{Abbreviations}

Andro: Andrographolide; H\&E: hematoxylin \& eosin; MVD: Microvessel density; IOD: integrated optical density.

\section{Acknowledgments}

The authors thank Xi-Min Zhang, Hui Chen, and Qiao-Bing Yuan for technical assistance.

\section{Funding}

This research was supported by the National Basic Research Program (No. 2010CB529703) from the Ministry of Science and Technology of China and the National Natural Science Foundation of China (No. 31271455, 31200896 and 31100852), the Medical Science Research Foundation of Guangdong Province, China (No. B2012181) and the Natural Science Foundation of Guangdong Province, China (S2012040007658). 


\section{Competing Interests}

The authors have declared that no competing interest exists.

\section{References}

1. Yao JC, Eisner MP, Leary C, Dagohoy C, Phan A, Rashid A, et al. Population-based study of islet cell carcinoma. Ann Surg Oncol. 2007; 14: 3492-500.

2. Schaefer R. Rare Cancers Europe: joining forces to tackle a common problem. Rare Tumors. 2012; 4: e24.

3. Bergers G, Javaherian K, Lo KM, Folkman J, Hanahan D. Effects of angiogenesis inhibitors on multistage carcinogenesis in mice. Science. 1999; 284: 808-12.

4. Hager JH, Hanahan D. Tumor cells utilize multiple pathways to down-modulate apoptosis. Lessons from a mouse model of islet cell carcinogenesis. Ann N Y Acad Sci. 1999; 887: 150-63.

5. Xia YF, Ye BQ, Li YD, Wang JG, He XJ, Lin X, et al. Andrographolide attenuates inflammation by inhibition of NF-kappa B activation through covalent modification of reduced cysteine 62 of p50. J Immunol. 2004; 173: 4207-17.

6. Iruretagoyena MI, Tobar JA, Gonzalez PA, Sepulveda SE, Figueroa CA, Burgos RA, et al. Andrographolide interferes with $\mathrm{T}$ cell activation and reduces experimental autoimmune encephalomyelitis in the mouse. J Pharmacol Exp Ther. 2005; 312: 366-72.

7. Shen YC, Chen CF, Chiou WF. Andrographolide prevents oxygen radical production by human neutrophils: possible mechanism(s) involved in its anti-inflammatory effect. Br J Pharmacol. 2002; 135: 399-406.

8. Zhou J, Zhang S, Ong CN, Shen HM. Critical role of pro-apoptotic Bcl-2 family members in andrographolide-induced apoptosis in human cancer cells. Biochem Pharmacol. 2006; 72: 132-44.

9. Rajagopal S, Kumar RA, Deevi DS, Satyanarayana C, Rajagopalan R. Andrographolide, a potential cancer therapeutic agent isolated from Andrographis paniculata. J Exp Ther Oncol. 2003; 3: 147-58.

10. Kawai T, Akira $\mathrm{S}$. The role of pattern-recognition receptors in innate immunity: update on Toll-like receptors. Nat Immunol. 2010; 11: 373-84.

11. Naugler WE, Karin M. NF-kappaB and cancer-identifying targets and mechanisms. Curr Opin Genet Dev. 2008; 18: 19-26.

12. Fukata M, Chen A, Vamadevan AS, Cohen J, Breglio K, Krishnareddy S, et al. Toll-like receptor-4 promotes the development of colitis-associated colorectal tumors. Gastroenterology. 2007; 133: 1869-81.

13. Rakoff-Nahoum S, Medzhitov R. Regulation of spontaneous intestinal tumorigenesis through the adaptor protein MyD88. Science. 2007; 317: 124-7.

14. Ikebe M, Kitaura Y, Nakamura M, Tanaka H, Yamasaki A, Nagai S, et al. Lipopolysaccharide (LPS) increases the invasive ability of pancreatic cancer cells through the TLR4/MyD88 signaling pathway. J Surg Oncol. 2009; 100: 725-31.

15. Chang I, Kim S, Kim JY, Cho N, Kim YH, Kim HS, et al. Nuclear factor kappaB protects pancreatic beta-cells from tumor necrosis factor-alpha-mediated apoptosis. Diabetes. 2003; 52: 1169-75.

16. Handa SS, Sharma A. Hepatoprotective activity of andrographolide from Andrographis paniculata against carbontetrachloride. The Indian journal of medical research. 1990; 92: 276-83.

17. Maciorowski Z, Delic J, Padoy E, Klijanienko J, Dubray B, Cosset JM, et al. Comparative analysis of apoptosis measured by Hoechst and flow cytometry in non-Hodgkin's lymphomas. Cytometry. 1998; 32: 44-50.

18. Wang LJ, Zhao $Y$, Han B, Ma YG, Zhang J, Yang DM, et al Targeting Slit-Roundabout signaling inhibits tumor angiogenesis in chemical-induced squamous cell carcinogenesis. Cancer Sci. 2008; 99: 510-7.

19. Xu H, He JH, Xiao ZD, Zhang QQ, Chen YQ, Zhou H, et al. Liver-Enriched Transcription Factors Regulate MicroRNA-122 That Targets CUTL1 During Liver Development. Hepatology. 2010; 52: 1431-42.

20. Sodir NM, Swigart LB, Karnezis AN, Hanahan D, Evan GI, Soucek L. Endogenous Myc maintains the tumor microenvironment. Genes Dev. 2011; 25: 907-16

21. Singh M, Johnson L. Using genetically engineered mouse models of cancer to aid drug development: an industry perspective. Clin Cancer Res. 2006; 12: 5312-28

22. Cheon DJ, Orsulic S. Mouse models of cancer. Annu Rev Pathol. 2011; 6: 95-119.

23. Politi $\mathrm{K}$, Pao $\mathrm{W}$. How genetically engineered mouse tumor models provide insights into human cancers. J Clin Oncol. 2011; 29: 2273-81.

24. Hanahan D. Heritable formation of pancreatic beta-cell tumours in transgenic mice expressing recombinant insulin/simian virus 40 oncogenes. Nature. 1985; 315: 115-22.

25. Kumar RA, Sridevi K, Kumar NV, Nanduri S, Rajagopal S. Anticancer and immunostimulatory compounds from Andrographis paniculata. J Ethnopharmacol. 2004; 92: 291-5.

26. Kim TG, Hwi KK, Hung CS. Morphological and biochemical changes of andrographolide-induced cell death in human prostatic adenocarcinoma PC-3 cells. In Vivo $2005 \cdot 19 \cdot 551-7$.
27. Wang LJ, Zhou X, Wang W, Tang F, Qi CL, Yang X, et al. Andrographolide inhibits oral squamous cell carcinogenesis through NF-kappaB inactivation. J Dent Res. 2011; 90: 1246-52

28. Kelly MG, Alvero AB, Chen R, Silasi DA, Abrahams VM, Chan S, et al. TLR-4 signaling promotes tumor growth and paclitaxel chemoresistance in ovarian cancer. Cancer Res. 2006; 66: 3859-68.

29. Wang AC, Su QB, Wu FX, Zhang XL, Liu PS. Role of TLR4 for paclitaxel chemotherapy in human epithelial ovarian cancer cells. Eur J Clin Invest. 2009; 39: $157-64$ 